\title{
Single-carrier fractional Fourier domain equalization system with zero padding for fast time-varying channels
}

\author{
Enqing Chen ${ }^{*}$ and CaiHong Chu
}

\begin{abstract}
Single-carrier frequency domain equalization (SC-FDE) has been shown to be an attractive transmission scheme for broadband wireless channels. However, its performance would degrade a lot if the channel is fast time-varying. In this paper, we analyzed the single-carrier fractional Fourier domain equalization (SC-FrFDE) system and applied it to the fast time-varying channel. It can solve the time-varying problem by selecting the optimal fractional Fourier transform order. To this SC-FrFDE system, its transmitter uses chirp-periodic circular prefix to eliminate ISI and this has an evident disadvantage that the receiver need to feedback the optimal fractional Fourier transform order to the transmitter through a feedback channel. To simplify the system, we propose to use zero padding (ZP) at the transmitter. There is already the overlap-add method as ZP in SC-FDE system. But the overlap-add method cannot be used in fast time-varying channels. Thus, we propose a new method as ZP. Simulation results show that our proposed method can significantly improve the system performance.
\end{abstract}

Keywords: Single-carrier fractional Fourier domain equalization; Chirp-periodic circular prefix; ZP; Fast time-varying channels

\section{Introduction}

Single-carrier frequency-domain equalization (SC-FDE) has been shown to be an attractive equalization scheme for broadband wireless channels which have very long impulse response memory [1]. SC radio modems with frequency domain equalization have very similar performance, efficiency, and low signal processing complexity advantages as OFDM, and in addition are less sensitive than OFDM to RF impairments [2]. So the SC-FDE system has the favorable advantages than the OFDM system. And this arises the use of SC modulation [2,3]. However, when met with fast-moving channels in broadband wireless communications, the performance of SC-FDE will degrade a lot because orthogonality among different subchannels at the receiver is destroyed and ICI arises.

The concept of fractional Fourier transform (FrFT) was first introduced by N. Wiener in 1929. Then it appears as a mathematical tool for solving quantum mechanics problems $[4,5]$, but this method did not gain

\footnotetext{
* Correspondence: enqingchen@gmail.com

School of Information Engineering, ZhengZhou University, HeNan Province, China
}

much attention. Until fractional Fourier transform was introduced in the field of image analysis in optics and signal processing by Mendlovic [6], Ozaktas [7], and W. Lohmann $[8,9]$ and had it attracted great attention. Much research has been done in the multicarrier system. FrFT was first applied in the multicarrier system by M. Martone in 2001 [10]. Afterwards, Tomaso Erseghe proposes to use affine Fourier transform to remove ICI in the multicarrier system under time-varying channel models in 2005 [11]. Sufficient research [12,13] has been done in the multicarrier system compared to the researches done in the single-carrier (SC) system. In 2012, the FrFT was applied in the SC system [14], but it mainly discusses the FrFT to cope with the deep fading problem. The detailed procedure of the system is not discussed. Based on this, we will discuss the SC-FrFDE system in detail and expand this system to the fast timevarying channels.

In SC-FrFDE, discrete fractional Fourier transform (DFrFT) and inverse discrete fractional Fourier transform (IDFrFT) replace fast Fourier transform (FFT) and inverse fast Fourier transform (IFFT) correspondingly. 
Because of the chirp periodicity of the sampled signals, it is needed to add a chirp-periodic circular prefix (chirp $\mathrm{CP}$ ) instead of the traditional cyclic prefix at the transmitter. By selecting the optimal fractional Fourier transform order, the chirp CP can remove the ISI as well as transform the linear convolution of the signal and the time-varying channel into fractional circular convolution. Thus, the SC-FrFDE system can cope with fast time-varying channels well.

The SC-FrFDE with chirp CP has an evident disadvantage that the calculation formula of chirp $\mathrm{CP}$ includes the transform order. So there needs a feedback channel between the transmitter and receiver to feedback the optimal fractional Fourier transform order. This will increase the complexity of the whole system. To simplify the system, we propose to use zero padding (ZP) at the transmitter. There is already the overlap-add method as ZP in SC-FDE system. But the overlap-add method cannot be used in fast time-varying channels. Thus, we propose a new method as ZP.

The rest of the paper is organized as follows: In section 2, we introduce the basic definitions of DFrFT and fractional circular convolution. In section 3, the SC-FrFDE system model with chirp CP is detailedly described and represent that this system can deal with fast time-varying channels. In section 4, the expression of ZP is given. And the principle of selecting the optimal fractional Fourier transform order is introduced in section 5. To show the validity of the proposed scheme, Monte Carlo simulation results are presented in section 6. Lastly, in section 7 , we make a conclusion.

\section{Preliminaries - discrete fractional Fourier transform and fractional circular convolution}

\subsection{Discrete fractional Fourier transform}

\subsubsection{Fractional Fourier transform}

$x(t)$ is the time domain signal. The $p$ th-order FrFT signal $X_{p}(u)$ is defined as

$$
X_{p}(u)=\left\{F_{p}(x(t))\right\}(u)=\int_{-\infty}^{+\infty} x(t) K_{p}(u, t) d t
$$

where

$$
\begin{aligned}
& K_{p}(u, t) \\
& =\left\{\begin{array}{lr}
A_{p} \cdot \exp \left[j \pi\left(u^{2} \cot a-2 u t \csc a+t^{2} \cot a\right)\right], a \neq n \pi \\
\delta(t-u), & a=2 n \pi \\
\delta(t+u), & a=(2 n \pm 1) \pi
\end{array}\right.
\end{aligned}
$$

In that expression, $A_{p}=\sqrt{1-j \cot a}, a=\frac{\pi}{2} p$. Since the FrFT is periodic with the period of 4 , the transform order $p$ can be limited to the interval of $[-2,2]$.

\subsubsection{Discrete time fractional Fourier transform (DTFrFT)}

The above is the FrFT of the continuous signal. However, in the SC system, the signal that we are going to deal with is discrete signal. So, it is required to know the DFrFT of the discrete signal.

First, let us see the definition of DTFrFT. Assuming $x(t)$ is analog, we can obtain the time domain sampled signal

$$
x_{s}(t)=x(t) \sum_{n=-\infty}^{\infty} \delta(t-n \Delta t)
$$

$\Delta t$ is the sampling interval of time domain. $\Delta t=1 / \mathrm{ml} /$ sr. Among which $\mathrm{ml}$ represents the modulation method. For example, $\mathrm{ml}=1$ corresponds to the BPSK modulation; $\mathrm{ml}=2$ corresponds to QPSK modulation. $\mathrm{sr}$ is the symbol transmission rate. In this paper, the symbol transmission rate is set to be $\mathrm{sr}=250,000 \mathrm{symbol} / \mathrm{s}$.

According to Equation 20 in [15], the DTFrFT of the sampled signals is shown as:

$$
\begin{aligned}
& X_{s p}(u)=\frac{1}{\Delta t} \cdot e^{j(\cot a / 2) u^{2}} \cdot\left[X_{p}(u) e^{-j(\cot a / 2) u^{2}} * \sum_{n=-\infty}^{\infty} \delta\left(u-n \frac{2 \pi \sin a}{\Delta t}\right)\right] \\
& =\frac{1}{\Delta t} \cdot e^{j(\cot a / 2) u^{2}} \cdot\left[\sum_{n=-\infty}^{\infty} X_{p}\left(u-n \frac{2 \pi \sin a}{\Delta t}\right) e^{-j(\cot a / 2)\left(u-n \frac{2 \pi \sin a}{\Delta t}\right)^{2}}\right]
\end{aligned}
$$

It is readily seen that the sampled signal is discrete in the time domain. But its FrFT is still analog in the FrFD. To get the DFrFT of the time domain discrete signal, it is required to sample in the FrFD.

\subsubsection{Discrete fractional Fourier transform (DFrFT)}

Assuming the sampling points in the time domain and the fractional Fourier domain are $N$ and $M$. Generally $M=N$. We can get $X_{p}(m):=X_{p}(m \Delta u), m=0,1, \ldots, N-1$. The FrFD sampling interval is $u=\frac{2 \pi \sin a}{\Delta t \times N}$.

Let $\boldsymbol{F}$ be the DFrFT matrix whose $(m, n)$ th element is

$$
\begin{aligned}
& F(m, n)=\rho_{1}^{m^{2}} \times W^{m n} \times \rho_{2}^{n^{2}}, m, n \in(0, N-1) \\
& \rho_{1}^{m^{2}}:=e^{j \cot \alpha \times \Delta u^{2} \times m^{2} / 2}, \rho_{2}^{n^{2}}:=e^{j \cot \alpha \times \Delta t^{2} \times n^{2} / 2} \\
& W:=e^{-j 2 \pi / N}
\end{aligned}
$$

Then the definition of DFrFT is

$$
\mathbf{X}_{p}=\boldsymbol{F} \cdot \boldsymbol{x}
$$

According to the FrFT sampling theorem, the discretization of time domain and FrFD respectively cause chirp periodic extension of the FrFD and the time domain. This process is given as follows:

$$
X_{p}(m-N) e^{-j \frac{j}{2} \cot \alpha(m-N)^{2} \Delta u^{2}}=X_{p}(m) e^{-j \frac{1}{2} \cot \alpha m^{2} \Delta u^{2}}
$$




$$
x(n-N) e^{j \frac{1}{2} \cot \alpha(n-N)^{2} \Delta t^{2}}=x(n) e^{j \frac{1}{2} \cot \alpha n^{2} \Delta t^{2}}
$$

This chirp periodicity is very important because it will be used in the guard interval (GI).

\subsection{Discrete fractional Fourier transform}

Let $x_{1}(n), x_{2}(n)$ be the time domain discrete finite sequences. Let us define $\underset{P}{\otimes}$ as the $p$ th-order fractional circular convolution with the period of $N$. According to [16], its mathematical expression is

$$
\begin{aligned}
& x_{1}(n) \underset{P}{\otimes} x_{2}(n)=\sqrt{\frac{\sin \alpha-j \cos \alpha}{N}} e^{-j \cot a n^{2} \Delta t^{2} / 2} . \\
& \sum_{i=0}^{N-1} x_{1}(i) e^{-j \cot a i^{2} \Delta t^{2} / 2} x_{2}((n-i))_{p, N} R_{N}(n) e^{-j \cot a(n-i)^{2} \Delta t^{2} / 2}
\end{aligned}
$$

In which $x_{2}((n))_{p, N}$ is the $p$ th-order chirp periodic extension sequence with period of $N$ and $R_{N}(n)$ is used to get the principal value interval.

$$
\begin{aligned}
& \text { The DFrFT of } x_{1}(n) \underset{P}{\stackrel{N}{\otimes}} x_{2}(n) \text { is } \\
& F_{p}\left[x_{1}(n) \underset{P}{\stackrel{N}{\otimes}} x_{2}(n)\right]=X_{1, p}(m) X_{2, p}(m) e^{-j \cot a m^{2} \Delta u^{2} / 2}
\end{aligned}
$$

This is the fractional circular convolution theorem.

\section{SC-FrFDE system}

The SC-FrFDE system is shown as Figure 1. After QPSK modulation, the current block contains $N$ samples $x(n)$, $n=0,1, \cdots, N-1$. In order to reduce the symbol interference, GI is needed to insert in. The cyclic prefix (CP) of $x(n)$ is not just the copy of its tail data but should have the chirp periodicity. This kind of CP is called chirp-periodic circular prefix; for simplicity, it is denoted as chirp CP. The signal being added chirp CP is expressed as

$$
\boldsymbol{x}_{\mathrm{cp}}=\left[x\left(-N_{c}\right), x\left(-N_{c}+1\right), \ldots, x(0), \ldots, x(N-1)\right]^{T}
$$

In this paper, $\{\cdot\}^{T},\{\cdot\}^{H},\{\cdot\}^{*}$ stand for transpose, conjugate transpose and conjugate. $N_{c}$ is the length of chirp CP. According to (10), the chirp CP is written as

$$
\begin{aligned}
x\left(-N_{c}+n\right) & =x\left(N-N_{c}+n\right) \cdot e^{j \frac{1}{2} \cot \alpha\left[\left(N-N_{c}+n\right)^{2}-\left(-N_{c}+n\right)^{2}\right] \Delta t^{2}}, n \\
& =0,1, \ldots, N_{c}-1
\end{aligned}
$$

To describe this process in matrix form, we finally get

$$
\boldsymbol{x}_{\mathrm{cp}}=\boldsymbol{T}_{\mathrm{cp}} \times \boldsymbol{x}
$$

in which

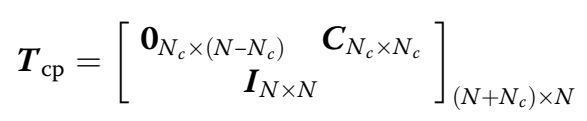

$0_{N_{c} \times\left(N-N_{c}\right)}$ is a $N_{c} \times\left(N-N_{c}\right)$ matrix with elements of zeroes. $\boldsymbol{I}_{N \times N}$ represents the identity matrix.

$$
\begin{aligned}
C_{N_{c} \times N_{c}} & =\operatorname{diag}\left[e^{j \frac{1}{2} \cot \alpha\left[\left(N-N_{c}+N\right)^{2}-\left(-N_{c}+n\right)^{2}\right] \Delta t^{2}}\right], n \\
& =0,1, \ldots, N_{c}-1
\end{aligned}
$$

After inserting chirp $\mathrm{CP}$, the signal is transmitted to the fast time-varying channels $\boldsymbol{H}$. It varies during one data block, and its time domain impulse response is $h(n, l)$ with length of $L$. Then the received signal is given by

$$
\boldsymbol{y}_{\mathrm{cp}}=\boldsymbol{H} \cdot \boldsymbol{x}_{\mathrm{cp}}+\boldsymbol{\gamma}_{\mathrm{cp}}
$$

$\gamma_{\mathrm{cp}}$ is the additive white Gaussian noise.

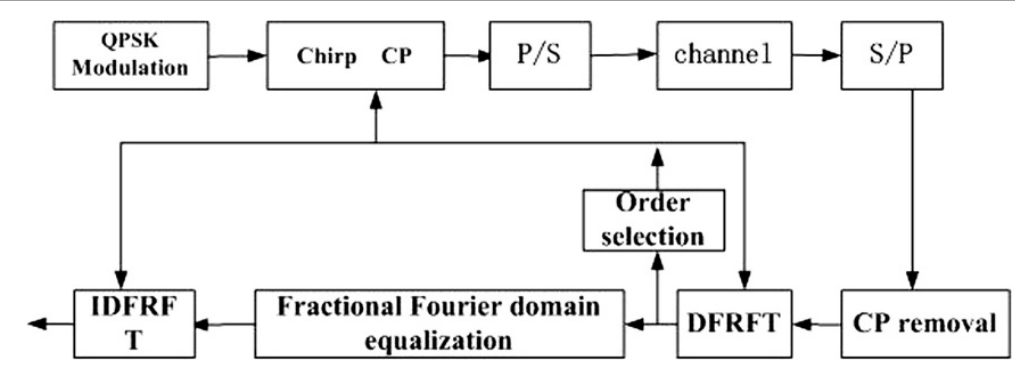

Figure 1 SC-FrFDE system. 


$$
\boldsymbol{H}=\left[\begin{array}{ccccc}
h\left(-N_{c}, 0\right) & 0 & \cdots & 0 & 0 \\
h\left(-N_{c}+1,1\right) & h\left(-N_{c}+1,0\right) & \cdots & 0 & 0 \\
\vdots & h\left(-N_{c}+2,1\right) & \ddots & \vdots & \vdots \\
h\left(-N_{c}+L-1, L-1\right) & \vdots & \ddots & \vdots & \vdots \\
0 & h\left(-N_{c}+L, L-1\right) & \vdots & \vdots & \vdots \\
\vdots & 0 & \vdots & 0 & \vdots \\
0 & \vdots & \vdots & h(N-2,0) & 0 \\
& 0 & \cdots & h(N-1,1) & h(N-1,0)
\end{array}\right]
$$

In SC-FrFDE system, the removal of chirp $\mathrm{CP}$ is the same with that in SC-FDE system and is expressed as follows:

$$
\begin{aligned}
& \boldsymbol{y}=\boldsymbol{R}_{c p} \cdot \boldsymbol{y}_{c p}=\boldsymbol{R}_{c p} \cdot \boldsymbol{H} \cdot \boldsymbol{T}_{c p} \cdot \boldsymbol{x}+\boldsymbol{\gamma}=\overline{\boldsymbol{H}} \cdot \boldsymbol{x}+\boldsymbol{\gamma} \\
& \boldsymbol{R}_{c p}=\left[\begin{array}{ll}
0_{N \times N_{c}} & \boldsymbol{I}_{N}
\end{array}\right] N \times\left(N+N_{c}\right) \\
& \boldsymbol{y}=[y(0), y(1), \ldots, y(N-1)]^{T}
\end{aligned}
$$

By adding and removing chirp $\mathrm{CP}$, the time domain channel matrix $\boldsymbol{H}$ is transformed to (23)

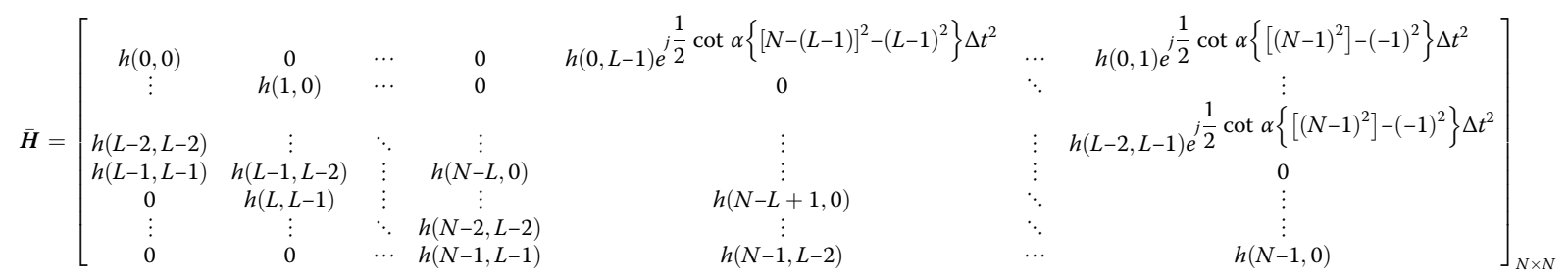

Through the process above, the linear convolution will be turned into fractional circular convolution of the signal and the equivalent channel response. Let us unfold (20) to explain this problem.

$$
\begin{aligned}
y(n)= & \sum_{l=0}^{L-1} h(n, l) x(N+n-l) \\
& \cdot e^{j \frac{1}{2} \cot \alpha[N+(n-l)]^{2} \Delta t^{2}-j \frac{1}{2} \cot \alpha(n-l)^{2} \Delta t^{2}} \\
& +\gamma(n) \quad 0 \leq n \leq L \\
y(n)= & \sum_{l=0}^{L-1} h(n, l) x(n-l)+\gamma(n) \quad L+1 \leq n \leq N-1
\end{aligned}
$$

Because of the chirp periodicity shown in (10), we can combine Equations 24 and 25 into one.

$$
\begin{aligned}
y(n) & =\sum_{l=0}^{L-1} h(n, l) \cdot x((n-l))_{p, N} \cdot R_{N}(n)+\gamma(n) \\
& =\sum_{l=0}^{N-1} h(n, l) \cdot x((n-l))_{p, N} \cdot R_{N}(n)+\gamma(n) \quad 0 \leq n \leq N-1
\end{aligned}
$$

Equation 26 indicates that by adding and removing chirp CP, the linear convolution is turned into circular convolution (It is not fractional circular convolution). It is the circular convolution about $h(n, l)$ and one chirp periodic extension sequence $x((n))_{p, N}$. 
Now let us turn this circular convolution into fractional circular convolution.

$$
\begin{aligned}
y(n) & =\sum_{l=0}^{N-1} h(n, l) \cdot x((n-l))_{p, N} \cdot R_{N}(n)+\gamma(n) \\
& =e^{\frac{-j \cot a \cdot n^{2} \Delta t^{2}}{2}} \sum_{l=0}^{N-1}\left[\frac{j \cot a \cdot n^{2} \Delta t^{2}}{2} h(n, l)\right] x((n-l))_{p, N} \cdot R_{N}(n)+\gamma(n) \\
= & e^{\frac{-j \cot a \cdot n^{2} \Delta t^{2}}{2}} \sum_{l=0}^{N-1}\left[\frac{j \cot a \cdot n^{2} \Delta t^{2}}{2} h(n, l) e^{\frac{-j \cot a \cdot(n-l)^{2} \Delta t^{2}}{2}} \frac{-j \cot a \cdot l^{2} \Delta t^{2}}{2}\right] \\
& e^{\frac{j \cot a \cdot l^{2} \Delta t^{2}}{2}} \cdot x((n-l))_{p, N} \cdot R_{N}(n) e^{\frac{j \cot a \cdot(n-l)^{2} \Delta t^{2}}{2}}+\gamma(n) \\
= & \sum_{l=0}^{\frac{-j \cot a \cdot n^{2} \Delta t^{2}}{2}} h_{p}(n, l) e^{\frac{j \cot a \cdot l^{2} \Delta t^{2}}{2}} \cdot x((n-l))_{p, N} \cdot R_{N}(n) e^{\frac{j \cot a \cdot(n-l)^{2} \Delta t^{2}}{2}}+\gamma(n)
\end{aligned}
$$

Define $\quad h_{p}(n, l)=h(n, l) e^{j \cot a \cdot n l \Delta t^{2}} e^{-j \cot a \cdot l^{2} \Delta t^{2}} \quad$ as the equivalent channel response. By selecting the proper FrFT order, $h_{p}(n, l)$ will be time-invariant or varies very slowly. In this circumstances, $h_{p}(n, l) \approx h_{p}(l)$; and Equation 27 can be seen as the fractional circular convolution. According to the fractional circular convolution theorem, we can get

$$
Y_{p}(k)=\left[e^{-j \cot a \cdot k^{2} \Delta u^{2} / 2} \cdot H_{p}(k)\right] \cdot X_{p}(k)+\widetilde{Y}(k)
$$

In which $H_{p}(k)=\sum_{l=0}^{L-1} h_{p}(l) \cdot e^{j \cot a \cdot l^{2} \cdot \Delta t^{2} / 2} \cdot e^{j \cot a \cdot k^{2} \cdot \Delta u^{2} / 2}$. $e^{-j \cdot 2 \pi l k / N} \cdot Y_{p}(k)$ and $X_{p}(k)$ is the fractional Fourier domain data of $y(n)$ and $x(n)$. The fractional circular convolution is very useful in the FrFD equalization.

Taking DFrFT of the received signal,

$$
\boldsymbol{Y}_{p}=\boldsymbol{F} \cdot \boldsymbol{y}=\boldsymbol{F} \cdot \overline{\boldsymbol{H}} \cdot \boldsymbol{F}_{-1} \cdot \boldsymbol{X}_{p}+\tilde{\boldsymbol{\gamma}}=\tilde{\boldsymbol{H}} \cdot \boldsymbol{X}_{p}+\tilde{\boldsymbol{\gamma}}
$$

$\boldsymbol{F}_{-1}$ is the IDFrFT matrix. $\boldsymbol{F}_{-1}=\boldsymbol{F}^{\boldsymbol{H}}$. According to (28), $\tilde{\boldsymbol{H}}$ is a diagonal matrix. And its diagonal elements are $e^{-j \cot a \cdot k^{2} \Delta u^{2} / 2} \cdot H_{p}(k)$. The final expression is

$$
\begin{aligned}
Y_{p}(k)= & \tilde{H}(k, k) \cdot X_{p}(k)+\tilde{\Upsilon}(k) \\
& =\left[e^{-j \cot a \cdot k^{2} \Delta u^{2} / 2} \cdot H_{p}(k)\right] \cdot X_{p}(k)+\tilde{\Upsilon}(k)
\end{aligned}
$$

It is assumed that we know the perfect knowledge of FrFD channel response $\tilde{\boldsymbol{H}}$. Then the FrFD data $\hat{X}_{p}$ can be obtained via

$$
\hat{X}_{p}(k) \approx Y_{p}(k) / \tilde{H}(k, k)
$$

At last, the FrFD data $\hat{X}_{p}$ is transformed to timedomain data $\hat{\boldsymbol{x}}$ by taking IDFrFT. The estimation of time-domain data is

$$
\hat{\boldsymbol{x}}=\boldsymbol{F}_{-1} \cdot \hat{\boldsymbol{X}}_{p}
$$

\section{Zero padding method}

If the transmitter uses chirp $\mathrm{CP}$ as guard interval, the receiver need to feedback the optimal fractional Fourier transform order to the transmitter through a feedback channel. It will increase the complicity of the system. To solve this problem, the transmitter could adopt ZP which has no information about the optimal fractional Fourier transform order. If the channel is slow time-varying, we could use the well-known overlap-add technique as ZP to make the circulant Toeplitz matrix [17]. But if the channel is fast time-varying, this method cannot be applied. First, let us show the invalid of overlap-add method under fast time-varying channels.

\subsection{The invalid of the traditional overlap-add method}

For simplicity, we will consider the overlap-add method under fast time-varying channels in the SC-FDE system. Just like channel matrix for zero padding in [17], we will 
observe the variations of the channel matrix $\boldsymbol{H}$. For simple case $N=4, N_{c}=2$. Channel matrix for zero padding in [17] can be rewritten as

Step 1:

\begin{tabular}{|c|c|c|c|c|c|c|c|c|c|c|c|c|c|}
\hline \multicolumn{14}{|c|}{$\boldsymbol{H}_{1}=\boldsymbol{H} \cdot \boldsymbol{T}_{z p}$} \\
\hline$h(0,0)$ & 0 & 0 & 0 & $h(0,0)$ & 0 & 0 & 0 & 0 & 0 & 1 & 0 & 0 & 0 \\
\hline$h(1,1)$ & $h(1,0)$ & 0 & 0 & $h(1,1)$ & $h(1,0)$ & 0 & 0 & 0 & 0 & 0 & 1 & 0 & 0 \\
\hline 0 & $h(2,1)$ & $h(2,0)$ & 0 & 0 & $h(2,1)$ & $h(2,0)$ & 0 & 0 & 0 & 0 & 0 & 1 & 0 \\
\hline 0 & 0 & $h(3,1)$ & $h(3,0)$ & 0 & 0 & $h(3,1)$ & $h(3,0)$ & 0 & 0 & 0 & 0 & 0 & 1 \\
\hline 0 & 0 & 0 & $h(4,1)$ & 0 & 0 & 0 & $h(4,1)$ & $h(4,0)$ & 0 & 0 & 0 & 0 & 0 \\
\hline 0 & 0 & 0 & 0 & 0 & 0 & 0 & 0 & $h(5,1)$ & $h(5,0)$ & 0 & 0 & 0 & 0 \\
\hline
\end{tabular}

Step 2:

$$
\begin{aligned}
& \overline{\boldsymbol{H}}=\boldsymbol{R}_{z p} \cdot \boldsymbol{H}_{1}
\end{aligned}
$$

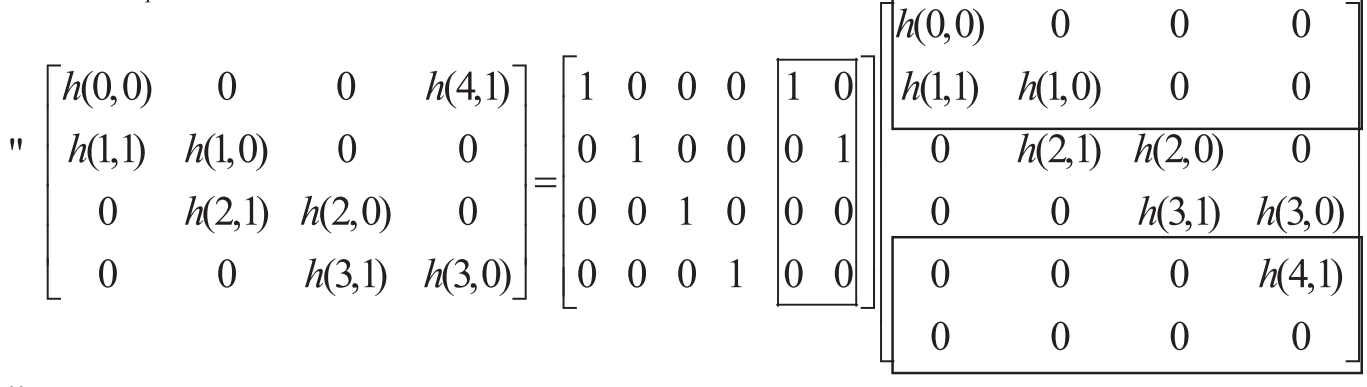

If the channel is invariant or varies slowly during one data block, $h(4,1) \approx h(0,1)$ and $\overline{\boldsymbol{H}}$ can be approximately seen as a circular matrix and thus can be diagonalized through the FFT/IFFT. But if the channel is fast time-varying, $\overline{\boldsymbol{H}}$ is obvious not a circular matrix and would not be diagonalized. This situation is exactly the same with the sC-FrFDE system. So the overlap-add method is not suitable for fast time-varying channels. We need to think of other ways to add ZP.

\subsection{Proposed method of zero padding \\ 4.2.1 Transmitter}

After QPSK modulation, the symbol is of size $N$. Assume $N_{c}$ is the length of GI. According to the knowledge of chirp CP, the fractional Fourier transform order $\alpha$ exists in the GI $x(n)=x(N+n) \cdot e^{j \frac{1}{2} \cot \alpha\left[(N+n)^{2}-n^{2}\right] \Delta t^{2}},-N_{c} \leq n \leq-1$ .If the last $N_{c}$ elements of $x(n)$ are $x(n)=0, N-N_{c} \leq n \leq$ $N-1$; then $x(n)=0,-N_{c} \leq n \leq-1$ which indicates that the transmitter did not include $\alpha$. To this method, the valid data length is $N-N_{c}$. So, the ZP method is based on the decrease of the data transmission efficiency. The data structure can be seen in process (1) Figure 2 . Then the data is fed into the zero padding modules. Assume the data being added ZP is of the length $N+$ $N_{c}$. And this process can be seen as (2) in Figure 2 . The expressions about the signal being added ZP is shown as

$$
\boldsymbol{x}_{\mathrm{zp}}=\left[0, \ldots, 0, x(0), \ldots, x\left(N-N_{c}-1\right), 0, \ldots, 0\right]^{T}
$$

To describe the process in matrix form, it is

$$
\begin{aligned}
& \boldsymbol{x}_{\mathrm{zp}}=\boldsymbol{T}_{\mathrm{zp}} \cdot \boldsymbol{x} \\
& \boldsymbol{T}_{\mathrm{zp}}=\left[\begin{array}{c}
\mathbf{0}_{N_{c} \times N} \\
\boldsymbol{I}_{N \times N}
\end{array}\right]_{\left(N+N_{c}\right) \times N}
\end{aligned}
$$

Then the signal is transmitted to the fast time-varying channel $\boldsymbol{H} . \boldsymbol{H}$ is shown in Equation 19. 


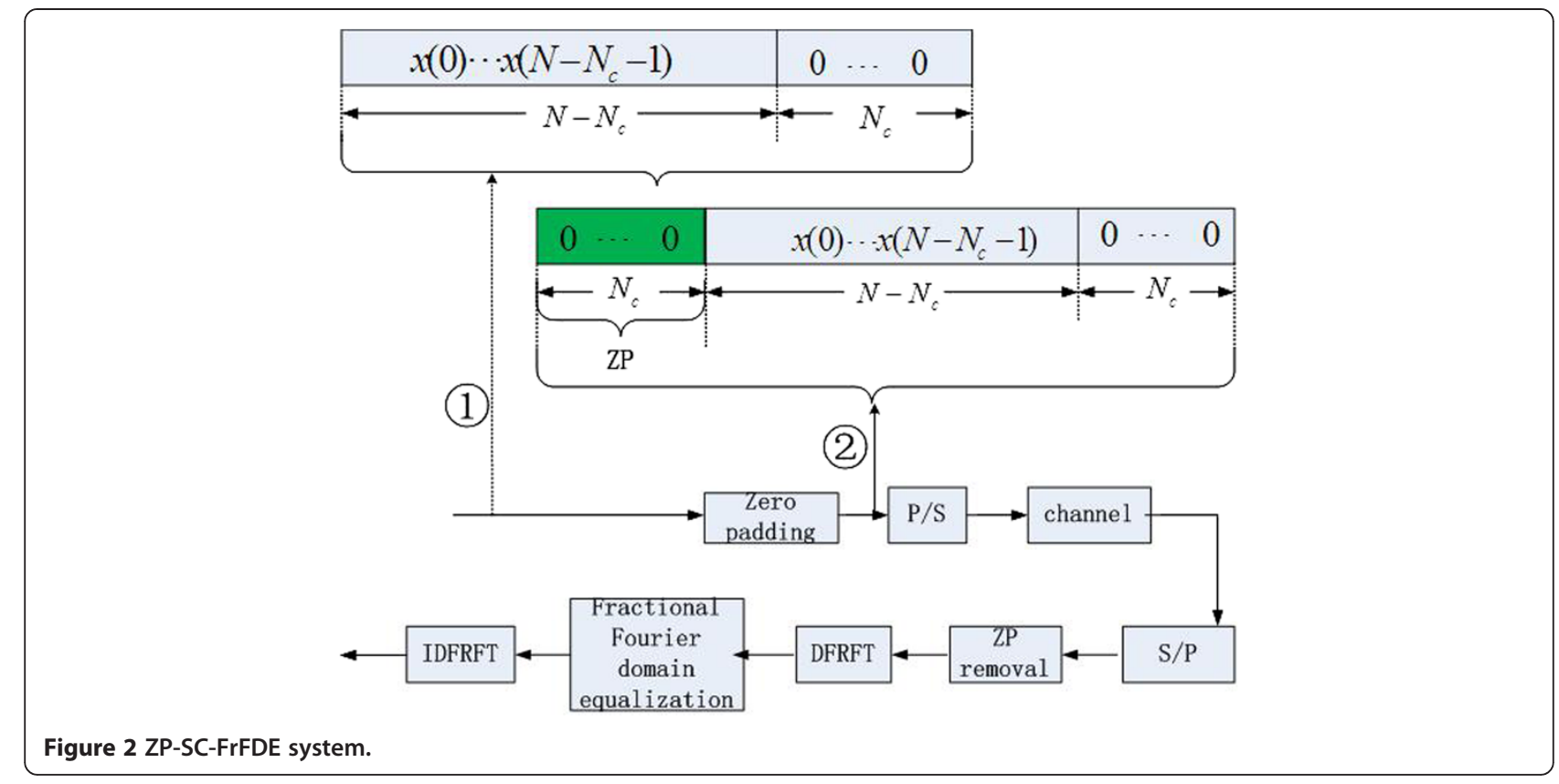

\subsubsection{Receiver}

The received signal is

$$
\boldsymbol{y}_{\mathrm{zp}}=\boldsymbol{H} \cdot \boldsymbol{x}_{\mathrm{zp}}+\boldsymbol{y}_{\mathrm{zp}}
$$

$\gamma_{\mathrm{zp}}$ is the additive white Gaussian noise.

The removal of GI is the same with the chirp CP-SCFrFDE system. After the received signals corresponding to the $\mathrm{ZP}$ are removed at the receiver, the $\mathrm{N}$-point data is expressed in the matrix form as

$$
\boldsymbol{y}=\boldsymbol{R}_{\mathrm{zp}} \cdot \boldsymbol{y}_{\mathrm{zp}}+\boldsymbol{\gamma}_{1}=\boldsymbol{R}_{\mathrm{zp}} \cdot \boldsymbol{H} \cdot \boldsymbol{T}_{\mathrm{zp}} \cdot \boldsymbol{x}+\boldsymbol{\gamma}_{1}=\overline{\boldsymbol{H}}_{1} \cdot \boldsymbol{x}+\boldsymbol{\gamma}_{1}
$$

where

$$
\boldsymbol{R}_{\mathrm{zp}}=\left[\begin{array}{ll}
0_{N_{c} \times N} & \boldsymbol{I}_{N \times N}
\end{array}\right]_{N \times\left(N+N_{c}\right)}
$$

To have a more detailed description of the problem, let us unfold Equation 39.

$$
\begin{aligned}
y(n) & =\sum_{l=0}^{L-1} h(n, l) x((n-l))_{N} \cdot R_{N}(n)+\gamma(n) \\
& =\sum_{l=0}^{L-1} h(n, l) \cdot x((n-l))_{p, N} \cdot R_{N}(n)+\gamma(n) \quad 0 \leq n \leq N-1
\end{aligned}
$$

$x((n-l))_{N}$ is a periodic signal with period $N$. It can be seen that Equation 42 is just the same with (26). And the following operation is very much like that of (27) which is the fractional circular convolution.

$$
\begin{aligned}
y(n)= & e^{\frac{-j \cot a \cdot n^{2} \Delta t^{2}}{2}} \sum_{l=0}^{N-1} h_{p}(n, l) e^{\frac{j \cot a \cdot l^{2} \Delta t^{2}}{2}} \\
& \cdot x((n-l))_{p, N} \cdot R_{N}(n) e^{\frac{j \cot a \cdot(n-l)^{2} \Delta t^{2}}{2}}+\gamma(n)
\end{aligned}
$$

$$
\begin{aligned}
& \overline{\boldsymbol{H}}_{1}=\boldsymbol{R}_{\mathrm{zp}} \cdot \boldsymbol{H} \cdot \boldsymbol{T}_{\mathrm{zp}} \\
& {\left[\begin{array}{cccccc}
h(0,0) & 0 & 0 & 0 & \cdots & 0 \\
\vdots & h(1,0) & 0 & \cdots & \ddots & \vdots \\
h(L-2, L-2) & \vdots & \ddots & \vdots & \vdots & 0 \\
h(L-1, L-1) & h(L-1, L-2) & \vdots & h(N-L, 0) & \vdots & 0 \\
0 & h(L, L-1) & \vdots & \vdots & \ddots & \vdots \\
\vdots & \vdots & \ddots & h(N-2, L-2) & \ddots & \vdots \\
0 & 0 & \cdots & h(N-1, L-1) & \cdots & h(N-1,0)
\end{array}\right]_{N \times N}}
\end{aligned}
$$




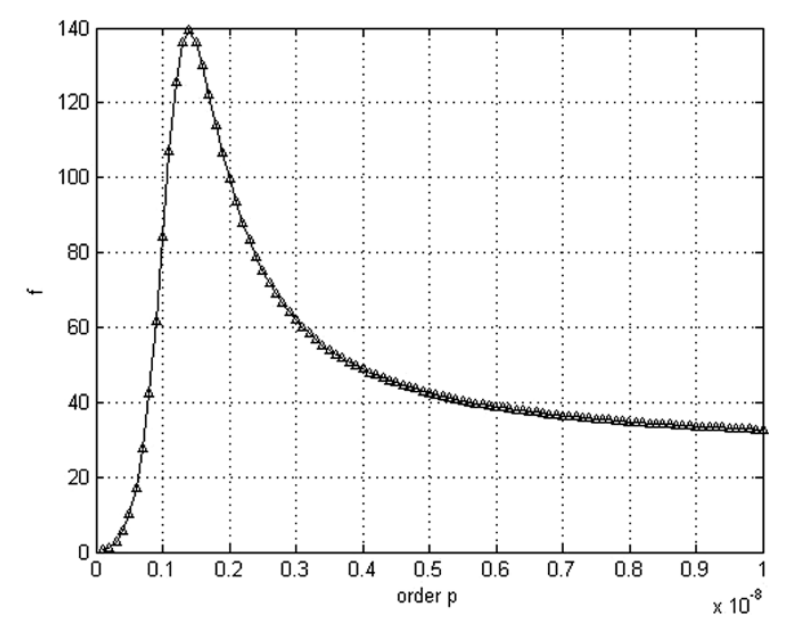

Figure 3 The target function versus the fractional Fourier transform order $p$ under channel 2 .

In Equation $43 h_{p}(n, l)=h(n, l) e^{j \cot a \cdot n l \Delta t^{2}} e^{-j \cot a \cdot l^{2} \Delta t^{2}}$. If $h_{p}(n, l) \approx h_{p}(l)$, then $Y_{p}(k)=e^{-j \cot a \cdot k^{2} \Delta u^{2} / 2} \cdot H_{p}(k) \cdot X_{p}(k)+$ $r(k)$

So the transmitted signal can be obtained by

$$
X_{p}(k) \approx Y_{p}(k) /\left(e^{-j \cot a \cdot k^{2} \Delta u^{2} / 2} \cdot H_{p}(k)\right)
$$

By taking IDFrFT, this signal will be transformed to time domain. When the signals get into the QPSK demodulation module, it is only essential for us to demodulate the first $N-N_{c}$ data (the last $N_{c}$ signals of the transmitting signal are zeros). And its BER performance is measured by its useful signals which are the first $N-N_{c}$ elements of the transmitted signals.

\section{Optimal fractional Fourier transform order}

In conventional SC-FDE system, the signal is modulated by the exponential bases. When met with fast-fading channels, the Fourier-domain matrix cannot be diagonalized. To solve this problem, we can think of the fast-fading channel as a channel with 'time-varying' frequency response. The optimal transmission/reception methodology should be able to 'diagonalize' nonstationary signals [10]. So, we propose to use the chirp basis instead of traditional exponential basis. By selecting the optimal fractional Fourier transform order, it can effectively cope with fast timevarying channels. In this section, we will give the principle that selects the optimal order.

First, let us write $\tilde{\boldsymbol{H}}$ in Equation 29 as this

$$
\tilde{\boldsymbol{H}}=\tilde{\boldsymbol{H}}^{u}+\tilde{\boldsymbol{H}}^{\mathrm{ICI}}
$$

Where $\tilde{\boldsymbol{H}}^{u}$ is made up of the diagonal elements of $\tilde{\boldsymbol{H}}$. $\tilde{\boldsymbol{H}}^{\mathrm{ICI}}$ represents the subchannel interference. By substitution of (45) into (29), we can get

$$
\boldsymbol{Y}_{p}=\left(\tilde{\boldsymbol{H}}^{u}+\tilde{\boldsymbol{H}}^{\mathrm{ICI}}\right) \cdot \boldsymbol{F} \cdot \boldsymbol{x}+\tilde{\boldsymbol{\gamma}}=\boldsymbol{s}_{u}+\boldsymbol{s}_{\mathrm{ICI}}+\tilde{\boldsymbol{\gamma}}
$$

$\boldsymbol{s}_{u}=\tilde{\boldsymbol{H}}^{u} \cdot \boldsymbol{F} \cdot \boldsymbol{x}$ and $\boldsymbol{s}_{\mathrm{ICI}}=\tilde{\boldsymbol{H}}^{\mathrm{ICI}} \cdot \boldsymbol{F} \cdot \boldsymbol{x}$ represent desired signal and the inter-carrier interference signal.

To get the optimal equalization performance, we need to try to increase the power of the desired signal and decrease the power of the inter-carrier interference signal. So our proposed method of choosing the optimal fractional Fourier transform order is selecting the fractional Fourier domain that makes the desired signal and the interference signal having the highest ratio. The target function is

$$
f_{\text {target }}(p)=\frac{E\left[\boldsymbol{s}_{u}{ }^{H} \boldsymbol{s}_{u}\right]}{E\left[\boldsymbol{s}_{\mathrm{ICI}}{ }^{H} \boldsymbol{s}_{\mathrm{ICI}}\right]}
$$

Because the transmitted signal is independent and identically distributed, so $E\left[\boldsymbol{x}^{H} \boldsymbol{x}\right]=\boldsymbol{I}$ (I is the identity matrix). It is already known that $E\left[\boldsymbol{F}^{H} \boldsymbol{F}\right]=\boldsymbol{I}$. The target function can also be written as

$$
f_{\text {target }}(p)=\frac{\left\|\tilde{\boldsymbol{H}}^{u}\right\|^{2}}{\left\|\tilde{\boldsymbol{H}}^{\mathrm{ICI}}\right\|^{2}}
$$

$\|\cdot\|$ is the Frobenius norm. The fractional Fourier transform order that carries signals should make (47) have the largest value, i.e.

$$
\left\{p_{\text {opt }}\right\}=\underset{p}{\operatorname{argmax}} f_{\text {target }}(p)
$$

This is the principle of choosing the optimal fractional Fourier transform order. In the engineering, if we search the order one by one, the computation complexity is very high. To degrade the complexity, we adopt the variable step searching method. At first, search $0<p<2$ at a big step to find the region that contains the optimal order. Then, we

Table 1 Simulation parameters of channels

\begin{tabular}{lccc}
\hline & Path & Delay $(\boldsymbol{\mu s})$ & Power $(\mathbf{d B})$ \\
\hline Channel 1 & 1 & 0 & 0 \\
Channel 2 & 2 & 6 & -5 \\
& 1 & 0 & 0 \\
& 2 & 5 & -3 \\
& 3 & 9 & -6 \\
& 4 & 12 & -9 \\
& 1 & 0 & 0 \\
& 2 & 2 & -3 \\
& 3 & 3 & -5 \\
& 4 & 5 & -10 \\
& 5 & 8 & -15 \\
& 6 & 11 & -20 \\
\hline
\end{tabular}




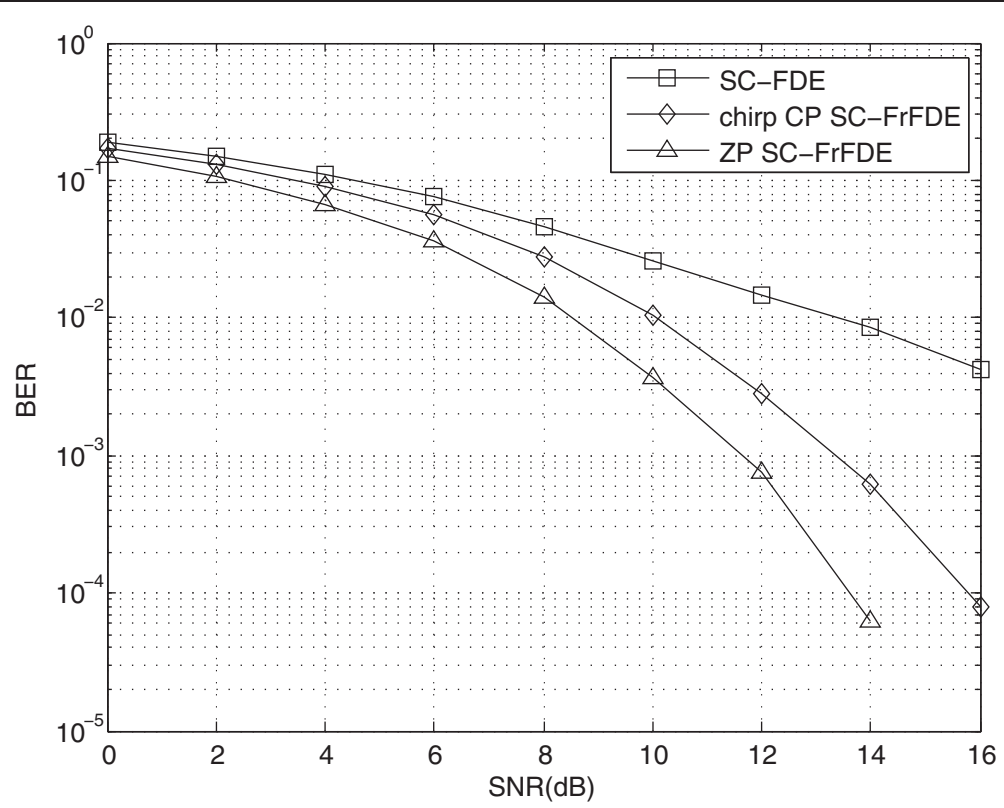

Figure 4 Comparison of the SC-FDE, chirp CP-SC-FrFDE and ZP-SC-FrFDE system under channel 2.

can search the optimal region with a small step to find the accurate optimal order.

Figure 3 shows the target function versus the fractional Fourier transform order. The channel parameters are shown in section 6. Without losing generality, the channel model is channel 2. It can be seen that there exists an optimal order to cope with the fast-fading channel.

\section{Simulation results}

In this section, we present the simulation results of the ZP-SC-FrFDE, compared with chirp CP-SC-FrFDE and the conventional cyclic prefixed SC-FDE. The performance of the system is measured by bit error rate (BER) with

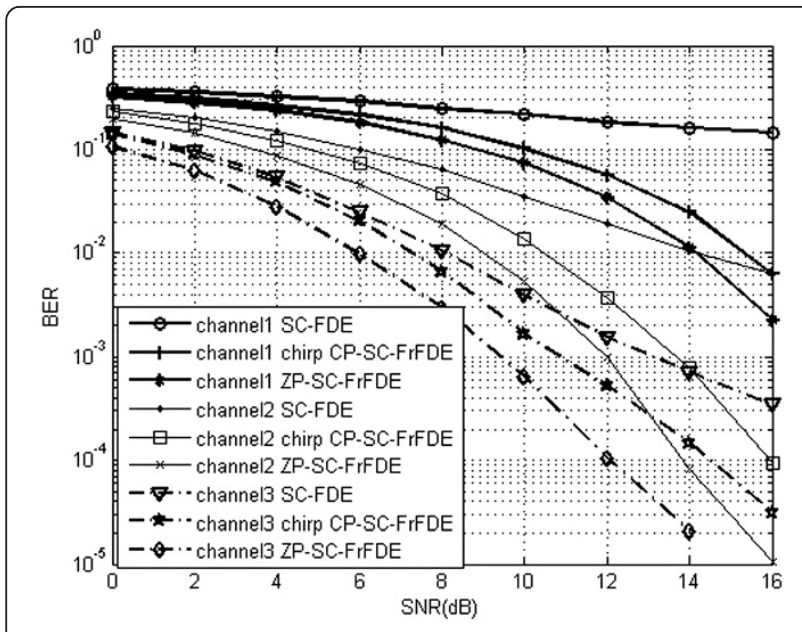

Figure 5 Comparison of SC-FDE, chirp CP-SC-FrFDE, and ZP-SC-FrFDE system under different channels.
1,000 realizations. We consider an uncoded single-carrier transmission with $N=64$ and QPSK constellation. The time domain sampling interval is $\Delta t=2 \times 10^{-6} \mathrm{~s}$. The length of the guard interval is 16 which is larger than the channel's maximum propagation delay.

The channels we used in the simulation are listed as Table 1.

Figure 4 compares the BER performance of the conventional SC-FDE system with the chirp CP-SC-FrFDE system and the ZP-SC-FrFDE system. Without loss of generality, the simulation is conducted under channel 2. The max Doppler shift is $1,000 \mathrm{~Hz}$. It can be seen that the chirp CP-SC-FrFDE system exceeds the conventional

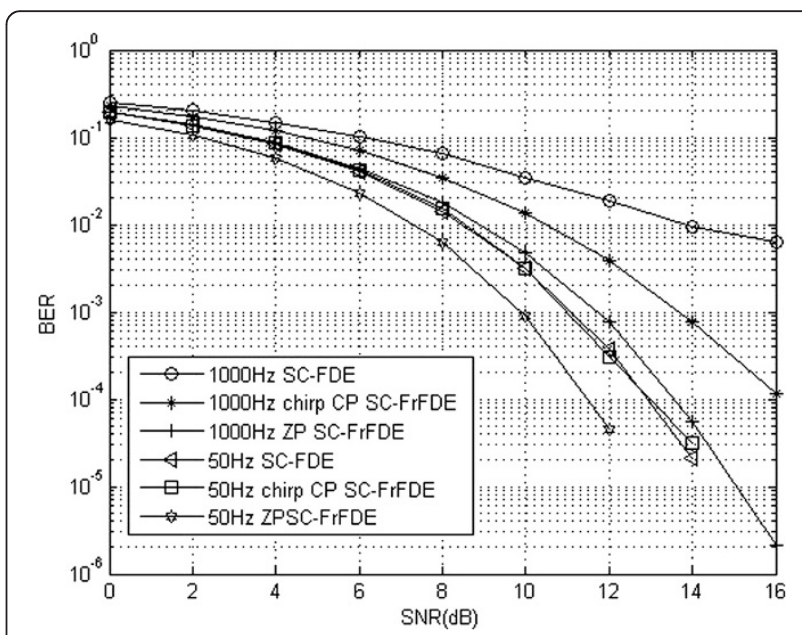

Figure 6 Comparison of SC-FDE, chirp CP-SC-FrFDE, and ZP-SC-FrFDE system under different Doppler frequency. 
SC-FDE system. And the ZP-SC-FrFDE system outperforms the chirp CP-SC-FrFDE system significantly. But this improvement is achieved on the sacrifice of decreasing its transmission efficiency.

Figure 5 gives the BER performance of the conventional SC-FDE system with the chirp CP-SC-FrFDE system and the ZP-SC-FrFDE system under different channels. The max Doppler shift is fixed at 1,000 Hz. To each channel, the fractional Fourier order is correspondingly the optimal. It is worthwhile noting that for each channel, the ZP-SC-FrFDE system performs the best BER performance. And the chirp CP-SC-FrFDE system can cope with fast-fading channels much better than the SC-FDE system.

Figure 6 compares the three systems under different Doppler frequencies. The FrFT orders in the simulation are correspondingly the optimal orders to each Doppler frequency. It can be seen that the ZP-SC-FrFDE system performs best among the three systems. If the max Doppler frequency is large, the chirp CP-SC-FrFDE system outperforms the SC-FDE system significantly. While the max Doppler frequency is small, the performance improvement will gradually decrease. When the Doppler shift is $50 \mathrm{~Hz}$, the curves of the chirp CP-SC-FrFDE almost coincide with the SC-FDE system. In this condition, the optimal fractional Fourier transform order $p \approx 1$. The DTFrFT would degenerate into DFT.

\section{Conclusions}

In this paper, we introduce the SC-FrFDE system to fast time-varying channels to solve the fast time-varying problem. Because this system needs to know the fractional Fourier transform order at the transmitter and there needs to be a feedback channel between the transceiver. The complexity forced us to find the zero padding method. The traditional overlap-add method cannot be used in fast time-varying channels. And we propose a new method to add ZP. The ZP-SC-FrFDE is discussed afterwards. Both of the two systems need to know the optimal fractional Fourier transform order, so we discussed the method of choosing the optimal fractional Fourier transform order. By using the optimal order, the fast time-varying channel matrix can be diagonalized in the FrFT domain. Simulation results show that the ZP-SC-FrFDE system outperforms the other two systems despite of the channel model and the max Doppler frequency.

\section{Competing interests}

The authors declare that they have no competing interests.

\section{Acknowledgements}

This work was supported partly by the National Natural Science Foundation of China under Grants No. 61201251, No.61172086, No.61210005 and No.61331021.

Received: 25 February 2014 Accepted: 16 April 2014

Published: 7 May 2014

\section{References}

1. YHR Zheng et al., Channel estimation for frequency-domain equalization of single-carrier broadband wireless communications. IEEE Trans. Vehicular Tech 58(2), 815-823 (2009)

2. D Falconer, SL Ariyavisitakul, A Benyamin-Seeyar, B Eidson, Frequency domain equalization for single-carrier broadband wireless systems. IEEE Commun. Mag. 40(4), 58-66 (2002)

3. MV Clark, Adaptive frequency domain equalization and diversity combinging for broadband wireless communications. IEEE J. Sel. Areas Commun 16(8), 1385-1395 (1998)

4. V Namias, The fractional order Fourier transform and its application in quantum mechanics. J. Inst. Math. Appl 25(3), 241-265 (1980)

5. AC McBride, FH Kerr, On Namias's fractional Fourier trans-forms. IMA J. Appl. Math. 39(2), 159-175 (1987)

6. D Mendlovic, HM Ozaktas, Fractional Fourier transforms and their optical implementation: I. J. Opt. Soc. Am.A. 10(9), 1875-1880 (1993)

7. HM Ozaktas, D Mendlovic, Fractional Fourier transforms and their optical implementation: II. J. Opt. Soc. Am. A 10(12), 2522-2531 (1993)

8. AW Lohmann, Image rotation, Wigner rotation, and the fractional order Fourier transform. J. Opt. Soc. Am. A 10(10), 2181-2186 (1993)

9. HM Ozaktas, Z Zalevsky, MA Kutay, The fractional Fourier Transform with Applications in Optics and Signal Processing (John Wiley \& Sons, New York, 2000)

10. M Martone, A multi-carrier system based on the fractional Fourier transform for time-frequency-selective channels. IEEE Trans. Commun. 49(6), 1011-1020 (2001)

11. T Erseghe, N Laurenti, V Cellini, A multicarrier architecture based upon the affine Fourier transform. IEEE Trans. Commun. 53(5), 853-862 (2005)

12. D Stojanovic, I Djurovic, BR Vojcic, Interference analysis of multicarrier systems based on affine Fourier transform. IEEE Trans. Wireless Commun. 8 (6), 2877-2880 (2009)

13. E Chen, R Tao et al., The OFDM system and equalization algorithm based on the fractional Fourier transform. Acta Electronica Sinica. 35(3), 409-414 (2007)

14. KW Huang, R Tao, Y Wang, Fractional Fourier domain equalization for single carrier broadband wireless systems. Sci. China 55(10), 2257-2268 (2012)

15. R Tao, B Deng, W-Q Zhang, Y Wang, Sampling and sampling rate conversion of band limitted signals in the fractional Fourier transform domian. IEEE Trans Signal Processing. 56(1), 158-171 (2008)

16. R Tao, B Deng, Y Wang, Fractional Fourier Transform and its Applications (Tsinghua University Press, Beijing, 2009)

17. K Sujin, L Keonkook, C Jongsub, K Joonhyuk, K Naesoo, S Park, Frequency domain equalizer using zero-padding for IR-UWB systems, IEEE International Conference on Ultra-Wideband, 2007, pp. 905-909

\section{doi:10.1186/1687-1499-2014-74}

Cite this article as: Chen and Chu: Single-carrier fractional Fourier domain equalization system with zero padding for fast time-varying channels. EURASIP Journal on Wireless Communications and Networking $20142014: 74$.

\section{Submit your manuscript to a SpringerOpen ${ }^{\circ}$ journal and benefit from: \\ - Convenient online submission \\ - Rigorous peer review \\ - Immediate publication on acceptance \\ - Open access: articles freely available online \\ - High visibility within the field \\ - Retaining the copyright to your article}

Submit your next manuscript at $>$ springeropen.com 